Karin Pittner

\title{
Regeln für die Bildung von freien Relativsätzen. Eine Antwort an Oddleif Leirbukt
}

\section{Einleitende Bemerkungen}

Freie Relativsätze geben eine Reihe von Rätseln auf. Wie schon der Terminus andeutet, handelt es sich hier um eine widersprüchliche Erscheinung, da ein Relativsatz sich per definitionem auf ein Element im Matrixsatz bezieht, dies bei „freien Relativsätzen" jedoch nicht der Fall ist. Sie üben im übergeordneten Satz selbständig eine Satzgliedfunktion aus. Andererseits drängt sich der Eindruck auf, daß ein Bezugselement im Matrixsatz weggelassen wurde, es sich mithin also um verkappte Attributsätze handelt.

Leirbukt hat sich kürzlich in einer empirischen Studie mit durch das Pronomen wer eingeleiteten Relativsätzen beschäftigt, und zwar vor allem im Hinblick auf die Frage, wann ein Bezugselement gesetzt wird bzw. nicht gesetzt wird (Leirbukt 1995). Hierzu haben eine Reihe von Grammatikern die Auffassung vertreten, daß ein Bezugselement im Matrixsatz nur dann fehlen kann, wenn das Relativpronomen die Kasusforderung des Matrixsatzes für die entsprechende Leerstelle erfüllt. So z. B. Schulz/ Griesbach: „Wenn das Relativpronomen und das Demonstrativpronomen gleiche Formen haben, so kann das Demonstrativpronomen ausfallen" (1978: 181; ganz ähnlich Eisenberg 1989: 350). Diese Regel für die Bildung von freien Relativsätzen (im folgenden „Übereinstimmungsregel") findet sich nicht nur in einigen gängigen Grammatiken des Deutschen, sondern wird auch von einigen generativen Grammatikern (unter dem Stichwort ,Matching") als konstitutiv für die Bildung von freien Relativsätzen im Deutschen angesehen.'

Es ist bekannt, daß die Übereinstimmungsregel sich auf die konkreten morphologischen Ka-

\footnotetext{
In der generativen Grammatik wird die Übereinstimmungsregel vertreten von Haider (1988: 47), im Anschluß daran auch von Bhatt (1990: 30). Engel (1990: 249) dagegen zieht auch die Möglichkeit nicht-übereinstimmender freier Relativsätze in Erwägung. Zahlreiche Belege dafür finden sich bei Paul (1920: 201 f.).

2 Beispiele bei Pittner (1991: 342), dort weitere Beispiele und Angabe der Fundstellen.
}

susformen bezieht. So ist der Satz (1 a) völlig in Ordnung, während (Ib) meist als ungrammatisch empfunden wird:

(1 a) Sie zerstört, was ihr in die Quere kommt.

(Ib) *Sie zerstört, wer ihr in die Quere kommt. $\mathrm{Da}$ in beiden Fällen der Matrixsatz einen Akkusativ fordert, das Relativpronomen jedoch nominativisch ist, zeigt sich, daß für die Übereinstimmungsregel nur die konkrete morphologische Kasusform entscheidend ist, nicht der ,abstrakte" Kasus. Es stört nicht, daß zwei verschiedene Kasus gefordert werden, wenn die Formen identisch sind. Daß diese Übereinstimmungsregel nicht generell gilt, habe ich in verschiedenen Arbeiten zu zeigen versucht (Bausewein 1990: 164 ff.; Bausewein 1991; Pittner 1991). Durch Leirbukts Arbeit wird dies bestätigt. Belege wie die folgenden sind relativ häufig $\mathrm{zu}$ finden und werden von vielen Sprechern auch nicht als ungrammatisch bewertet:

(2) Wem die ätherischen Öle zu scharf sind, greift zu der leicht salzig schmeckenden Solezahnpasta. (DAT statt NOM)

(3) Jeder muß tun, wofür er bestimmt ist. (PP statt AKK)

(4) Erforscht wird, wofür's Geld gibt. (PP statt NOM) 2

Damit stellt sich dann aber die Frage, unter welchen Bedingungen die Übereinstimmungsregel außer Kraft gesetzt werden kann, denn es ist unzweifelhaft, daß die meisten freien Relativsätze tatsächlich dieser Regel genügen.

\section{Die Hierarchieregel}

Anhand von Informantenbefragungen und Belegsammlungen bin ich zu dem Ergebnis gekommen, daß bestimmte Abweichungen häufiger auftreten und eher toleriert werden als andere. Die Ergebnisse habe ich in folgender Regel zusammengefaßt:

(5) Bei einem Kasuskonflikt zwischen dem vom Matrixverb geforderten Kasus K1 und dem vom Verb im freien Relativsatz geforderten Kasus K2 kann K1 unrealisiert bleiben, wenn K1 K2 auf folgender Hierarchie vorangeht: 
(KH) Nominativ $>$ Akkusativ $>$ Dativ /Präpositionalkasus.

Die Wirkung dieser und ähnlicher Kasushierarchien wurde auch schon für andere Sprachen beobachtet, allerdings bleibt der theoretische Status dieser Hierarchie weitgehend unklar (McCreight 1987; Harbert 1983). Eine mögliche Erklärung liegt darin, daß es sich um eine Hierarchie „morphologischer Markiertheit" handelt, wobei der Nominativ im Sinne der Markiertheitstheorie als unmarkierter Kasus gelten kann, da er am häufigsten durch ein Nullaffix realisiert wird. Die Markiertheit der anderen Kasus ergibt sich dann aus der Anzahl der Formen, die sich von der Nominativform unterscheiden. Legt man das Paradigma der Relativpronomen ( $d$ - und w-Pronomen) zugrunde, so ist nach dem Nominativ der Akkusativ der am wenigsten markierte Kasus, gefolgt von Dativ und Genitiv.

(6) $d$-Pronomen

Mask Neutr Fern Plural

NOM der das die die

AKK den das die die

DAT dem dem der denen

GEN dessen dessen deren deren/derer

w-Pronomen

Mask Neutr

NOM wer was

AKK wen was

DAT wem -

GEN wessen wessen

Leirbukt bringt nun eine Reihe von Gegenbeispielen zu der Beobachtung, daß der NOM durch den AKK ersetzt werden kann, dies umgekehrt jedoch nicht gilt. Er führt (vor allem literarische) Beispiele dafür an, daß wer ohne Bezugselement auch dann stehen kann, wenn das Matrixverb einen AKK, in zwei Fällen auch einen DAT fordern würde:

(7a) Er läuft durch den Saal und küßt ab, wer gerade in seinem Tanzschritt sitzt.

(7b) Man spricht von Leistungsdruck, zu dem man gezwungen sei, einem Joch, das man abschütteln müsse, man spricht von der Leistungsgesellschaft, die nur gelten lasse, wer sich unterwerfe. $^{1}$

Anhand von Beispielen wie diesen kommt Leirbukt zu dem Schluß, daß für den von ihm untersuchten Teilbereich die Hierarchieregel nicht ausschlaggebend ist.

Dazu ist Verschiedenes anzumerken. Zunächst einmal kann nicht jeder Beleg als Beweis gelten. Hier wäre noch durch Befragung von In- formanten abzusichern, inwieweit diese Sätze noch als grammatisch bewertet werden. Nach meiner Erfahrung halten die meisten Sprecher des Deutschen solche Sätze nicht mehr für grammatisch. Außerdem sagt die Hierarchieregel die möglichen Abweichungen, deren Häufigkeit und Grammatikalität präzise voraus. Wenn die Kasushierarchie eine Hierarchie morphologischer Markiertheit ist und sich die Markiertheit eines Kasus an der Zahl vom Nominativ distinktiver Formen messen läßt, dann ist klar, daß der Unterschied zwischen NOM und AKK minimal ist, da es im Paradigma der Relativpronomen genau jeweils eine distinktive AKK-Form gibt. Die Diskrepanz NOM/AKK sollte also weniger gravierend sein als die Diskrepanz von z.B. NOM/DAT oder NOM/ GEN, und dies scheint auch tatsächlich der Fall zu sein. Bei Leirbukt finden sich nur zwei Beispiele für NOM statt DAT, doch eine ganze Reihe für NOM statt AKK.2 Generell soll mit der Hierarchieregel nicht gesagt sein, daß entsprechende Gegenbeispiele nie auftreten, sondern daß sie auf jeden Fall seltener sind und von den Sprechern auch als schlechter beurteilt werden als die Fälle von Nicht-Übereinstimmung, die durch die Hierarchieregel erfaßt werden. Daneben sind jedoch andere Faktoren zu berücksichtigen, die die Bildung von freien Relativsätzen ermöglichen.

\section{Andere Faktoren}

Wie Leirbukt bemerkt, kann die „Setzung und Nicht-Setzung des Korrelats" nicht durch eine einzige Regel erfaßt werden, da verschiedene Faktoren eine Rolle spielen. Auch die Stellung des Relativsatzes spielt eine entscheidende Rolle. Ein Bezugselement kann nur in Ausnahmefällen vor einem durch wer eingeleiteten Relativsatz stehen, was auch für die anderen Kasusformen dieses Pronomens gilt, nicht jedoch für das neutrale Gegenstück was. Nach einem durch wer eingeleiteten Relativsatz kann ein Korrelat jederzeit auftreten.

Die einheitliche Verwendung des Begriffs „Korrelat" sowohl für ein vorausgehendes wie für ein nachfolgendes Element sollte aber nicht darüber hinwegtäuschen, daß es hier einen grundlegenden syntaktischen Unterschied gibt. Geht ein „Korrelat" voran, so handelt es sich um eine Bezugs-NP für den Relativsatz, der somit kein freier, sondern ein attributiver Relativsatz ist. Steht das Element dagegen danach, so 
handelt es sich um eine Linksversetzungskonstruktion3 mit dem „Korrelat" als wiederaufnehmender Proform. Der Relativsatz bleibt in diesem Fall ein freier Relativsatz. Die nachfolgende Proform kann nicht Kern einer Attributkonstruktion sein, da ein attributiver Relativsatz im Deutschen nicht seiner Bezugs-NP vorangehen kann. Freie Relativsätze in Linksversetzungsposition brauchen generell nicht der Kasusforderung des Matrixsatzes zu genügen, da diese durch die Proform erfüllt wird.

(8) Wer das nicht einsieht, dem kann man nicht helfen.

Diese undifferenzierte Verwendung des Begriffs „Korrelat" führt m. E. Leirbukt auch dazu, Blatz' Beobachtung, daß bei Relativsätzen vor Frage- und Ausrufesätzen generell ein Korrelat stehen müsse, pragmatisch zu erklären, unter Rekurs auf verschiedene Illokutionstypen. 4

(9) Wer das Feuer fürchtet, ist der ein tapferer Soldat?

(10) Wer nach der Heimat wandert, wie rüstig der schreiten kann! (Beispiele bei Blatz 1900: 867)

Hier sind die Möglichkeiten einer rein syntaktischen Erklärung aber längst noch nicht ausgeschöpft. Satz (9) ist ein Entscheidungsfragesatz mit Verberststellung, der kein Vorfeld aufweist. Ebenso weist Satz (10) als selbständiger Verbendsatz nach traditioneller Auffassung kein Vorfeld auf.5 Daher bleibt in beiden Sätzen für den freien Relativsatz nur die Linksversetzungsposition mit Wiederaufnahme durch ein Pronomen. Ein Ausweichen in die Pragmatik scheint hier nicht nötig, es sei denn, man schreibt auch

1 Vgl. Leirbukt (1995); dort auch Angaben über die Belegstellen.

2 Vgl. auch die Belegsammlung bei Paul (1920). Sie enthält insgesamt 29 Beispiele für nicht-übereinstimmende Relativsätze, die der Hierarchieregel genügen. Von den 5 von der Hierarchieregel abweichenden Beispielen weisen 4 NOM statt AKK auf, 1 NOM statt DAT.

3 Als ,Linksversetzung" (,left dislocation") werden Konstruktionen bezeichnet, in denen eine Topik-Konstituente am linken Satzrand außerhalb der eigentlichen Satzstruktur auftritt und durch eine Proform im Satz wieder aufgenommen wird.

4 Leirbukt (1995). Allerdings scheint bei Entscheidungsfragesätzen ein Korrelat nicht unbedingt notwendig zu sein, da es auch Verbzweitfragesätze (gekennzeichnet allein durch die steigende Intonation) gibt.

5 In stärker generativ beeinflußten topologischen Analysen stehen $w$ - und rf-Pronomina nicht als linkes Klammerelement, sondern im Vorfeld, so z. B. bei Grewendorf/ Hamm/ Sternefeld (1987: 215); bei beiden Analysen existiert jedoch kein Vorfeld, in dem der Relativsatz stehen könnte. schon den Verbstellungstypen einen pragmatischen Charakter zu, was das Gebiet der Syntax aber zu sehr einschränken würde.

Da Leirbukt beobachtet, daß das Korrelat zu wer-Sätzen meist nicht auftreten kann, wenn ihm der Relativsatz nachfolgen würde, also aus dem freien ein attributiver Relativsatz würde mit Wechsel vom w-Pronomen zum (/-Pronomen, schränkt er seine Fragestellung auf die Korrelatsetzung nach vorausgehenden Relativsätzen ein. Dies ist im Sinne seiner Untersuchung der durch wer eingeleiteten Relativsätze durchaus konsequent, man begibt sich jedoch damit der Möglichkeit, die Regeln für die Bildung von freien Relativsätzen allgemein zu untersuchen. Wenn nämlich das vorangehende bzw. nachfolgende „Korrelat" jeweils bei bestimmten Kasuskonstellationen gesetzt werden $\mathrm{mu} \beta$, ist das ein deutlicher Hinweis darauf, da $\beta$ die Rektionsverhältnisse im Matrixsatz die Setzung dieses Pronomens erzwingen.

Neben der Hierarchieregel lassen sich noch andere Faktoren beobachten, die eine Abweichung von der Übereinstimmungsregel begünstigen. Bei den von Leirbukt zitierten abweichenden Beispielen, in denen wer steht, obwohl der Matrixsatz einen AKK (oder DAT) erfordert, steht der freie Relativsatz sehr häufig extraponiert im Nachfeld. Allgemein scheinen Abweichungen eher bei Relativsätzen im Nachfeld toleriert werden zu können. Ich habe das dahingehend zu erklären versucht, daß Relativsätze, die auf den Matrixsatz folgen, den Hörer/ Leser nicht mehr durch ein nicht in den Kasusrahmen des Matrixverbs passendes Relativpronomen irreführen können, da das Matrixverb und seine Leerstellen bereits bekannt sind (Bausewein 1990: 179). Dies ist eine funktionale Erklärung, die auf den Verarbeitungsaufwand und eventuelle Reanalyseprozesse Bezug nimmt, die bei nicht übereinstimmenden Relativsätzen im Vorfeld und Mittelfeld anfallen können. Leirbukt hält diese Erklärung für unzureichend, da sie nicht alle Fälle abdecken kann. Die von ihm genannten Gegenbeispiele sind aber großteils bereits durch die Hierarchieregel erfaßt. So z. B. der konstruierte Satz ${ }^{*}$ Er vertraut, wen er kennt. Es scheint generell nicht möglich zu sein, einen vom Matrixverb geforderten Dativ durch einen Akkusativ zu ersetzen, was durch die Hierarchieregel erfaßt ist. Auch das folgende Beispiel stellt kein Problem dar (Leirbukt 1995):

(11) Zum unangefochtenen Stadtadel zählt, wessen 
Familie gescheiterweise schon seit Generationen auf der Insel lebt...

Hier entfällt im Matrixsatz ein NOM, der Relativsatz wird durch ein attributives Genitivpronomen eingeleitet. Der Genitiv ist selbstverständlich ein stark markierter Kasus, der jederzeit das Wegfallen des Nominativs ermöglicht, nicht jedoch umgekehrt. Überdies sind Relativsätze wie in (1 1) auch gut im Vorfeld möglich, sie haben also mit Extraposition nichts zu tun.

Ein weiteres Argument gegen den „Extrapositions-Faktor" sieht Leirbukt darin, daß in früheren Sprachstufen des Deutschen Relativsätze ohne Korrelat relativ frei gebildet werden konnten. Hier bezieht er sich auf Groos/van Riemsdijk (1981), die davon ausgehen, daß im Neuhochdeutschen freie Relativsätze im Gegensatz zu früheren Sprachstufen nur nach der Übereinstimmungsregel gebildet werden. Der Unterschied zwischen den verschiedenen Sprachstufen liegt jedoch nicht darin, da $\beta$ freie Relativsätze im Alt- und Mittelhochdeutschen nicht der Übereinstimmungsregel genügen mußten, im Neuhochdeutschen dagegen schon. Vielmehr konnte das Relativpronomen in früheren Sprachstufen bei Kasusdiskrepanz den vom Matrixsatz geforderten Kasus tragen (sogenannte „Attraktion"), was heute ausgeschlossen ist.'

Extraposition muß als ein zusätzlicher Faktor gesehen werden, der die Bildung von nichtübereinstimmenden und der Hierarchieregel nicht genügenden freien Relativsätzen begünstigt. Bestimmte Beispiele, die sich an der Grenze des Akzeptablen bewegen, werden dann eher akzeptiert, wenn der Relativsatz im Nachfeld steht. Extraposition scheint also vor allem dann begünstigend zu wirken, wenn der Kasuskontrast ohnehin nicht stark ausgeprägt ist, wie zwischen NOM und AKK. Es ist auf keinen Fall ein $\mathrm{Zu}-$ fall, daß beinahe alle von Leirbukts MW-Sätzen, die nicht der Hierarchieregel genügen, extraponiert stehen. Wie auch immer eine Erklärung dafür letztlich aussieht. Extraposition ist einer der beteiligten Faktoren, die die Bildung nichtübereinstimmender freier Relativsätze begünstigen. Daß gewisse Beispiele wegen anderer Faktoren so schlecht sind, daß auch die Extraposition dies nicht mehr ausgleichen kann, spricht nicht dagegen. Vielmehr zeigt es, daß verschiedene Faktoren gegeneinander abzuwägen sind.

Daneben zeigt sich in Leirbukts Beispielmaterial ein weiterer Faktor, der über die tatsächli- chen Rektionsverhältnisse hinwegtäuscht und damit das Auftreten dieser MW-Sätze begünstigt, die weder von der Übereinstimmungsregel noch von der Hierarchieregel erfaßt werden. Bei seinen Beispielen (28a, b) und (29a) handelt es sich durchweg um die zweiten Konjunkte in einer NP, die meist das Komplement einer Präposition ist. Ein Beispiel soll hier zur Illustration genügen (Leirbukt 1995):

(12) ...irgendwie gab es einem wieder Vertrauen zu diesem Dumela und wer alles dahinterstand! An anderer Stelle bemerkt Leirbukt (1995), daß ansonsten inakzeptable freie Relativsätze (die der Hierarchieregel nicht genügen) durch Elemente zwischen dem regierenden Matrixverb und dem freien Relativsatz abgepuffert werden können, so daß die Verletzung der Hierarchieregel weniger auffällt.

(13) Sagen Sie das bitte Frau Schwarzkopf, Herrn Müller, Herrn Schmidt und wen sie sonst noch treffen.

(14) *Sagen Sie das bitte, wen Sie sonst noch treffen.

Auch hier zeigt sich, daß alles, was über die tatsächlichen Rektionsverhältnisse hinwegtäuscht - und dazu gehört auch die Distanz zwischen Matrixverb bzw. Matrixpräposition und freiem Relativsatz -, Abweichungen begünstigt.

Die Verletzung der Hierarchieregel wird also bei größerer Distanz weniger augenfällig, die Hierarchieregel selbst kann jedoch durch diesen „Puffer-Faktor" nicht wegerklärt werden. Steht der Relativsatz neben dem Matrixverb, ist der Unterschied deutlich sichtbar. (15a) wird durchweg als schlechter beurteilt als (15b):

(15a) *Er vertraut, wen er kennt. (AKK statt DAT)

(15b) Er lädt ein, wem er zu Dank verpflichtet ist. (DAT statt AKK)

Es bleiben einige wenige von Leirbukts Beispielen (1995), bei denen der freie Relativsatz nicht der Hierarchieregel genügt und auch nicht extraponiert steht oder durch dazwischenstehende Elemente abgepuffert ist:

(16a) Schwerer Blütenduft liegt zwischen den Mauern und läßt, wer hier lebt, den Geruch bitterster Armut gleich nebenan vergessen.

(16b) Das ist der einzige Weg, wie wir auf die

1 So auch Pittner (1995), wo das Wirken der Kasushierarchie bei der Bildung von Relativsätzen in anderen Sprachstufen des Deutschen beschrieben wird, insbesondere auch bei der sog. Attraktion, die im Althochdeutschen und auch im Mittelhochdeutschen noch möglich war; vgl. hierzu Harbert(1989: 138-149). 
Schnelle das Geld auftreiben, um uns, wer immer auch hinter ihm her ist, vom Hals schaffen zu können.

Wenn man einmal davon absieht, daß diese

Sätze nur noch schwerlich als grammatisch gelten können, kann man die Frage stellen, warum in diesen Sätzen eine Konstruktion mit freiem Relativsatz gewählt wurde. Die Frage kann man auch so stellen: Was wären die Alternativen zu einem freien Relativsatz an diesen Stellen? Es wären jedenfalls ziemlich schwerfällige und unhandliche Konstruktionen, was uns zu der Frage bringt, warum überhaupt freie Relativsätze existieren und zu welchen Zwecken sie eingesetzt werden. Ein wichtiger Grund liegt darin, daß sie die Möglichkeit bieten, generalisierende Aussagen zu machen und dabei schwerfällige Wiederholungen von gleich- oder ähnlichlautenden Pronomen zu vermeiden. Freie Relativsätze drücken meist eine indefinite oder generalisierende Referenz aus, deren generalisierender Charakter durch Zusätze wie auch immer und sonst noch verstärkt werden kann und mit anderen sprachlichen Mitteln kaum gleichwertig zu erreichen ist. Es ist auffällig, daß bei den meisten von Leirbukts Beispielen solche generalisierenden Zusätze auftreten.

Generalisierende Aussagen können also einer der Gründe sein, sich für die Konstruktion mit freiem Relativsatz zu entscheiden, was sich ja auch daran zeigt, daß freie Relativsätze sehr häufig in Sprichwörtern zu finden sind (Wer rastet, rostet. Wer wagt, gewinnt. Singe, wem Gesang gegeben!). Daneben können stilistische Faktoren wirksam sein, da freie Relativsätze eine kompaktere Ausdrucksmöglichkeit darstellen als Formulierungen mit derjenige, der oder alle, die etc. Bei einer Entscheidung für einen freien Relativsatz müssen allerdings verschiedene andere Faktoren berücksichtigt werden, die oben angesprochen wurden. Überwiegen jedoch die Vorteile des freien Relativsatzes und die Nachteile von Alternativen, wird man sich eventuell trotz der anderen Faktoren für diese Konstruktion entscheiden.

\section{Schlußbemerkung}

Die meisten freien Relativsätze, die nicht der Übereinstimmungsregel genügen, werden durch die Hierarchieregel in (5) erfaßt. Falls man den nicht von dieser Regel erfaßten Beispielen noch eine (marginale) Akzeptabilität zusprechen kann, werden auch diese Abweichungen noch durch die Hierarchieregel erfaßt. Wenn die Kasushierarchie die zunehmende morphologische Markiertheit der Kasus widerspiegelt, dann kann vorausgesagt werden, daß bestimmte Kasuskontraste deutlicher sind als andere. Insbesondere ist der Kontrast NOM vs. AKK sehr schwach, da nur eine distinktive Form in den entsprechenden Paradigmen auftritt. Abweichungen von der Hierarchieregel werden durch weitere Faktoren begünstigt. Dazu gehören die Extrapositionsstellung des freien Relativsatzes sowie Stellung des freien Relativsatzes als zweites Konjunkt, wodurch die Rektionsverhältnisse undeutlich werden.

In dem Zusammenwirken verschiedener Faktoren liegt jedoch keine Besonderheit der freien Relativsätze. Auch für andere grammatische Phänomene wurde in letzter Zeit ein Zusammenspiel verschiedener Faktoren mit unterschiedlicher Gewichtung beobachtet, so z. B. von Jacobs (1988) zur Abfolge der Satzglieder im Mittelfeld oder von Pafel (1993) bei der Bestimmung des Skopus von Quantoren.

Auch wenn noch nicht alle Fragen geklärt sind und insbesondere das Gewicht der einzelnen Faktoren noch ungeklärt ist, ist jetzt der Weg frei für weitere empirische Untersuchungen und Erklärungsansätze in einem Gebiet, auf das der Blick durch die (zu) normative und der Sprachwirklichkeit nicht gerecht werdende Grammatikschreibung lange verstellt war.

\section{Literatur}

Bausewein, Karin (1990): Akkusativobjekt, Akkusativobjektsätze und Objektsprädikate im Deutschen. Untersuchungen zu ihrer Syntax und Semantik. Tübingen.

Bausewein, Karin (1991): Haben kopflose Relativsätze tatsächlich keine Köpfe? In: G. Fanselow/ S. Felix (Hg.), Strukturen und Merkmale syntaktischer Kategorien. Tübingen, 144-158.
Bhatt, Christa (1990): Zur Syntax der deutschen Nominalphrase. Tübingen.

Blatz, Friedrich (1900): Neuhochdeutsche Grammatik mit Berücksichtigung der historischen Entwicklung der deutschen Sprache. Reprint Hildesheim/ New York 1970.

Eisenberg, Peter (21989): Grundriß der deutschen Grammatik. Stuttgart. 
Engel, Ulrich (1990): Deutsche Grammatik. Heidelberg.

Grewendorf, Günther/ Hamm, Fritz/Sternefeld, Wolfgang (1987): Sprachliches Wissen. Frankfurt a. M.

Groos, Anneke/Riemsdijk, Henk van (1981): Matching effects in free relatives: A parameter of core grammar. In: A. Belletti u. a. (Hg.), Theory of markedness in generative grammar. Pisa, 171 bis 216.

Haider, Hubert (1988): Zur Syntax der deutschen Nominalphrase. In: Zeitschrift für Sprachwissenschaft 7,32-59.

Harbert, Wayne (1983): On the Nature of the Matching Parameter. In: The Linguistic Review 2, 237-284.

Harbert, Wayne (1989): Case Attraction and the Hierarchization of Case. In: Proceedings of the Eastern States Conference on Linguistics 6, 138-149.

Jacobs, Joachim (1988): Probleme der freien Wortstellung im Deutschen. In: Sprache und Pragmatik 1, 8-37.

Leirbukt, Oddleif (1995): Über Setzung und Nichtset- zung des Korrelats bei Relativsätzen mit wer im heutigen Deutsch. In: H. Popp (Hg.), Deutsch als Fremdsprache. An den Quellen eines Faches. München.

McCreight, Kate (1987): Case hierarchies: the evidence from free relatives. In: Papers from the 23rd Annual Meeting ofthe Chicago Linguistic Society. Chicago, 259-267.

Pafel, Jürgen (1993): Scope and Word Order. In: J.Jacobs/A, von Stechow/Th. Vennemann (Hg.), Syntax. Ein internationales Handbuch, 1. Halbband. Berlin, 867-880.

Paul, Hermann (1920): Deutsche Grammatik. Band 4. Halle.

Pittner, Karin (1991): Freie Relativsätze und die Kasushierarchie. In: E. Feldbusch/R. Pogarell/ C. Weiß (Hg.), Neue Fragen der Linguistik. Akten des 25. Linguistischen Kolloquiums in Paderborn. Band 1. Tübingen, 341-347.

Pittner, Karin (1995): The case of German relatives. In: The Linguistic Review 12/3.

Schulz, Dora/Griesbach, Heinz ( $\left.{ }^{11} 1978\right)$ : Grammatik der deutschen Sprache. München.

\section{Die Monotonieeigenschaften der Intensivierwörter im Deutschen. Ein Beitrag zur Textsemantik des Deutschen (II)1}

\section{Intensivierer aus dem abschwächenden In- tensivierungsbereich \\ 4.1 Einführung}

Aufgrund Biedermanns (1969: 169) Einteilung rechnet man (wie etwa van Os 1986:230) die folgenden Intensivierer zum abschwächenden Bereich : etwas, ein wenig, ein klein wenig, ein bißchen, bißchen, (ein) bissei, ein kleines bißchen, geringfügig, schwach, leicht, halbweg(s), mäßig, mit Maßen, leidlich, leise und vag. Die Intensivierer mehr oder weniger, mehr oder minder, irgendwie, gewissermaßen, sozusagen schwanken ihrer Bedeutung nach zwischen dem gemäBigten und dem schwachen Intensivierungsbereich. Nach van Os (1985: 231) sind die meisten dieser Intensivierer positiv polar, weil sie mit der Negation unverträglich sind. Die einzige
Ausnahme bildet ein bißchen, das aber unter Negation keine Umkehrung des Skalenwertes bewirkt - ganz im Gegenteil: gerade das nicht. nicht ein bißchen bedeutet ja ,gar nichts", ist somit als verstärkte Negation zu betrachten.

Es sollen von diesen Intensivierern hier etwas, ein wenig, ein bißchen, mäßig und mehr oder weniger auf ihre Monotonieeigenschaften überprüft werden.

4.2 Das Indefinitpronomen ,etwas"

$\mathrm{Zu}$ etwas werden im Dudenwörterbuch zwei Bedeutungen genannt. Die erste Bedeutung lautet „ein bißchen, ein wenig", die zweite ,aliquid".

Der erste Teil dieses Beitrags erschien in DaF 3/1995 S.153-160. 